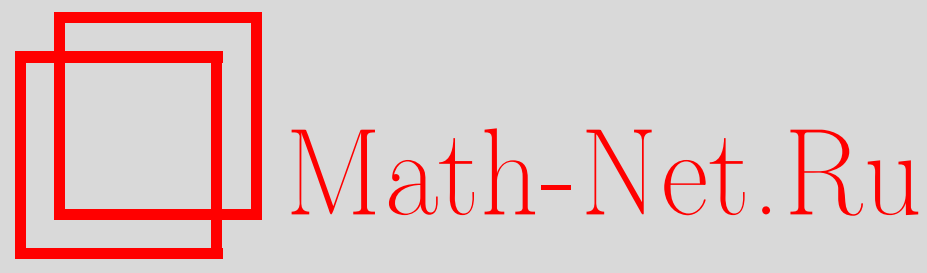

Г. М. Молчан, Линейные задачи для дробного броуновского движения: групповой подход, Теория вероятн. и ее примен., 2002, том 47, выпуск 1, 5970

DOI: https://doi.org/10.4213/tvp2985

Использование Общероссийского математического портала MathNet.Ru подразумевает, что вы прочитали и согласны с пользовательским соглашением

http://www.mathnet.ru/rus/agreement

Параметры загрузки:

IP : 54.89 .56 .158

26 апреля 2023 г., 18:37:18

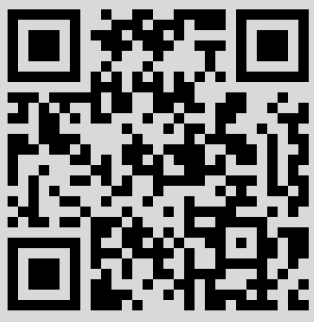




\title{
ЛИНЕЙНЫЕ ЗАДАЧИ ДЛЯ ДРОБНОГО БРОУНОВСКОГО ДВИЖЕНИЯ: ГРУППОВОЙ ПОДХОД ${ }^{1)}$
}

\begin{abstract}
Для дробного броуновского движения (fBm) рассмотрены задача прогноза с отрезка, каноническое представление $\mathrm{fBm}$ через белый шум на отрезке и их обратная связь, а также формула Гирсанова. Общий подход к задачам основан на инвариантности $\mathrm{fBm}$ относительно дробно-линейных преобразований времени. Этот подход практически исключает решение интегральных уравнений и объясняет эффективность решения перечисленных задач для $\mathrm{fBm}$.
\end{abstract}

Ключевые слова и фразы: дробное броуновское движение, прогноз, формула Гирсанова.

\section{1. Введение}

Гауссовский процесс $w_{H}(t), w_{H}(0)=0$, со структурной функцией

$$
\mathbf{E}\left|w_{H}(t)-w_{H}(s)\right|^{2}=|t-s|^{2 H}, \quad 0<H<1,
$$

принято называть дробным броуновским движением (fBm). Он является простейшим примером случайного процесса с непрерывными траекториями, обладающего свойствами аффинной инвариантности:

$$
\left\{w_{H}(\Lambda t+a)-w_{H}(a), t \in \mathbf{R}^{1}\right\} \stackrel{\mathrm{d}}{=}\left\{\Lambda^{H} w_{H}(t), t \in \mathbf{R}^{1}\right\}, \quad \Lambda \neq 0,
$$

и удаленного взаимодействия: при $H \neq \frac{1}{2}$ корреляции приращений $w_{H}$ убывают степенным образом, т.е.

$$
\mathbf{E}\left(w_{H}(t+1)-w_{H}(t)\right)\left(w_{H}(s+1)-w_{H}(s)\right) \sim c|t-s|^{2 H-2}, \quad|t-s| \rightarrow \infty .
$$

Благодаря этим свойствам процесс $w_{H}$ стал необычайно популярен в последнее десятилетие. Круг приложений (финансовая математика, телекоммуникационные сети, физические и биологические модели и т.п.)

\footnotetext{
*МИТП РАН, Варшавское шоссе, 79, корп. 2, 113556 Москва, Россия; е-mail: molchan@mitp.ru

1) Часть лекции, прочитанной на школе в Финляндии: Nauvo/Nagu Workshop, 19-22.8.2000. Работа выполнена при поддержке Российского фонда фундаментальных исследований (грант № 99-01-00314) и ISTC (грант № 1293-99).
} 
сейчас настолько расширился, что объективный обзор литературы вряд ли возможен. Наряду с броуновским движением $\left(H=\frac{1}{2}\right)$ процесс $w_{H}$ стал базовым для построения стохастического исчисления. При этом понадобилось решение простейших линейных задач для fBm. Сюда мы включаем прогноз $w_{H}$ по данным на отрезке, каноническое представление $\mathrm{fBm}$ через белый шум и их обратную связь на полуоси $t \geqslant 0$, т.е.

$$
w_{H}(t)=\int_{0}^{t} K(t, s) d w(s) \quad \text { и } \quad w(t)=\int_{0}^{t} K^{-}(t, s) d w_{H}(s),
$$

где $w(s)=w_{1 / 2}(s)$, а также формулу Гирсанова. Все эти задачи были решены в 60-е годы и заново переоткрыты в 90-е. Причина в том, что ключевое слово «fВm» утвердилось недавно, затруднив доступ к информации о ранних работах по процессу (1) в поисковых системах. К сожалению, повторные «элементарные» решения указанных задач базируются, как и прежде, на формальной аналитике в решении интегральных уравнений первого рода со степенным разностным ядром. Ниже показано, что эффективность решения линейных задач объясняется инвариантностью прирацений $w_{H}(t)$ относительно дробно-линейных преобразований времени. Любая упомянутая задача на отрезке редуцируется к тривиальной для $\mathrm{fBm}$ задаче на полупрямой. Это обстоятельство не было осознано ни в теоретико-вероятностных работах, ни в работах по интегральным уравнениям [1], [2]. В то же время групповой подход был использован автором в [3], но никогда не публиковался, за исключением финальных результатов. Повышенное внимание к fBm стимулировало написание данной заметки, включая исторические замечания.

История вопроса. В 1940 г. А.Н. Колмогоров выделил два важных свойства развитой турбулентности: локальную однородность (стационарность приращений в случае процессов) и автомодельность. В связи с этим появился новый вероятностный объект: случайный процесс со степенной структурной функцией (1), см. [4], [5]. В 1953 г. А. М. Яглом и М.Г. Пинскер совместно [6], [7] развили для процессов со стационарными $n$-ми приращениями спектральную теорию и теорию прогнозирования. В 1954-1955 гг. К. Ито [8] и И. М. Гельфанд [9] создали общую теорию обобщенных случайных функций, значительно расширив рамки локально-однородных случайных функций. Аппарат обобщенных процессов является естественным средством при решении линейных проблем fBm (см. ниже). Г. Хант упоминает спектральное представление для fBm в 1951 г. [10], а первые прогнозные формулы для $w_{H}$, основанные на этом представлении, появились в работе А. М. Яглома в 1955 г. [11]. Прогноз строился по данным $w_{H}$ на полуоси $(-\infty, a)$, $a \geqslant 0$. Одновременно М. Г. Крейн опубликовал работу [1] о новом методе решения интегральных уравнений. Она содержит практически все элементы, необходимые для канонического представления $w_{H}$ через белый 
шум на отрезке, хотя и требует дополнительного перевода на вероятностный язык. В 1967 г. С. В. Григорьев [12], отталкиваясь от работы А. М. Яглома [11], нашел обшее решение задачи прогноза $\mathrm{fBm} \mathrm{c} \mathrm{произ-}$ вольного отрезка. В 1968 г. Мандельброт и Ван Несс [13] предложили линейное представление приращений $w_{H}$ через белый шум на всей оси, т.е. представление вида

$$
w_{H}(t)-w_{H}(s)=c \int\left[(t-x)_{+}^{H-1 / 2}-(s-x)_{+}^{H-1 / 2}\right] d w(x),
$$

где $a_{+}=\mathbf{1}_{a>0}$. Эти же авторы предложили называть $w_{H}$ дробным броуновским движением. В 1968 г. автором получено решение всех перечисленных линейных задач для $\mathrm{fBm} ;$ в [14] решение основано на идеях М.Г. Крейна [1], а в [3] используется дополнительно групповой подход, излагаемый ниже. В [14] совместно с Ю.И. Голосовым рассмотрен также вопрос об эквивалентности гауссовых мер, одна из которых отвечает $\mathrm{fBm}$ на отрезке.

Новая волна интереса к линейным задачам для fBm возникла в последние пять лет и отражена в работах [15]-[18].

\section{2. Групповой подход}

2.1. Дробный шум. Пусть $\xi(t)=w_{H}^{\prime}(t), 0<H<1$, - слабая производная $w_{H}$, т.е. обобщенный процесс в смысле Гельфанда-Ито $\xi(\varphi)=$ $-\int w_{H}(t) \varphi^{\prime}(t) d t, \varphi \in K$, где $K$ - пространство финитных бесконечно дифференцируемых функций с обычной топологией Л. Шварца [19]. Из спектрального представления корреляционной функции $w_{H}$

$$
\mathbf{E} w_{H}(t) w_{H}(s)=c_{H}^{-1} \int_{-\infty}^{\infty}\left(e^{i t \lambda}-1\right)\left(e^{-i s \lambda}-1\right)|\lambda|^{-2 H-1} d \lambda
$$

с константой $c_{H}=-4 \Gamma(-2 H) \cos \pi H$ следует, что

$$
\mathbf{E}|\xi(\varphi)|^{2}=c_{H}^{-1} \int|\widehat{\varphi}(\lambda)|^{2}|\lambda|^{-\mu} d \lambda=c_{H}^{-1}\|\varphi\|_{\mu}^{2}, \quad \mu=2 H-1,
$$

где $\widehat{\varphi}$ - преобразование Фурье функции $\varphi$. Поэтому замыкание множества $\{\xi(\varphi), \varphi \in K\}$ случайных величин в $L^{2}$-метрике, $\|\xi\|^{2}=\mathbf{E} \xi^{2}$, изоморфно гильбертову пространству $H_{\mu}$, полученному замыканием $K$ в метрике $\|\cdot\|_{\mu}^{2}$. Пространство $H_{\mu}$ является естественной областью определения процесса $\xi$. В частности, $\xi\left(\chi_{(0, t)}\right)=w_{H}(t)$, где $\chi_{\Delta}$ - характеристическая функция отрезка $\Delta$.

Пусть $T$ - линейная операция в $H_{\mu}$, а $T^{*}$ - операция, формально сопряженная с $T$ относительно билинейной формы $\int \varphi(t) \psi(t) d t$. Тогда $(T \xi)(\varphi):=\xi\left(T^{*} \varphi\right)$ есть новый обобшенный процесс, если отображение $T^{*}: K \rightarrow H_{\mu}$ непрерывно. 
Обобщенный гауссовский процесс $\xi_{\mu}$ с корреляционным функционалом $\|\varphi\|_{\mu}^{2}$ определен для всех $\mu<1$. В рассматриваемом случае, $0<H<1$, параметр $|\mu|<1$; при $\mu=-1 \xi_{\mu}$ соответствует еще одному процессу Колмогорова [1], [2] с корреляционной функцией $\ln |t-s|^{-1}$.

Утверждение 1. Прочесс $\xi_{\mu}, \mu<1$, инвариантен относительно дробно-линейных преобразований оси времени:

$$
T \xi_{\mu}:=\xi_{\mu}\left(\frac{a t+b}{c t+b}\right)\left|\frac{c t+d}{\Delta}\right|^{\mu-1} \stackrel{\mathrm{d}}{=} \xi_{\mu}(t),
$$

аде $\Delta^{2}=|a d-b c| \neq 0$ и $T$ понимается в слабом смысле (см. выне).

Д о к а з а т е л ь с т в о. Группа дробно-линейных преобразований порождается сдвигами $t \rightarrow t+a$, растяжениями $t \rightarrow \Lambda t$ и инверсией $t \rightarrow 1 / t$. Инвариантность интересующего нас процесса $\xi_{\mu},|\mu|<1$, относительно аффинных преобразований является прямым следствием (2). При $|\mu|<1$ проверка аффинной инвариантности $\xi_{\mu}$ не составляет труда. Покажем, что процесс $\xi_{\mu}$ инвариантен относительно инверсии Мёбиуса:

$$
\xi_{\mu}(t) \stackrel{\mathrm{d}}{=} \xi_{\mu}\left(t^{-1}\right)|t|^{\mu-1}:=J_{\mu} \xi_{\mu}
$$

В силу гауссовости процесса $\xi_{\mu}$ достаточно показать, что сопряженная операция $J_{\mu}^{*} \varphi(t)=-\varphi(1 / t)|t|^{-\mu-1}$ является унитарной в пространстве $H_{\mu}$. Из (3) имеем

$$
\|\varphi\|_{\mu}^{2}=\int\left|(-i \lambda)^{n} \widehat{\varphi}(\lambda)\right|^{2}|\lambda|^{-\mu-2 n} d \lambda .
$$

Выберем целое $n$ так, чтобы $0<\mu+2 n \leqslant 2$. Используя преобразование Фурье обобщенной функции $|x|^{p}$ (см. [19]) и применяя равенство Парсеваля к (6), получим при $\mu \neq 1-2 n$

$$
\|\varphi\|_{\mu}^{2}=c(n) \iint|t-s|^{2 n+\mu-1} \varphi^{(n)}(t) \varphi^{(n)}(s) d t d s .
$$

При $\mu=1-2 n$ ядро надо заменить на $\ln |t-s|$. Проверим изометричность $J_{\mu}^{*}$ при $\mu \neq 1-2 n$. Без потери общности можно считать, что $|\mu|<1$. Полагая $n=1$, имеем для $\varphi \in K, \operatorname{supp} \varphi \in \mathbf{R}^{1} \backslash\{0\}$ :

$$
\left\|J_{\mu}^{*} \varphi\right\|_{\mu}^{2}=c(1) \iint|t-s|^{\mu+1} d\left[\varphi\left(t^{-1}\right)|t|^{-\mu-1}\right] d\left[\varphi\left(s^{-1}\right)|s|^{-\mu-1}\right] .
$$

После замен $t \rightarrow 1 / t, s \rightarrow 1 / s$ и раскрытия дифференциалов получим

$$
\begin{array}{r}
\left\|J_{\mu}^{*} \varphi\right\|_{\mu}^{2}=\|\varphi\|_{\mu}^{2}+c(1)(\mu+1) \iint|t-s|^{\mu+1}\left[\varphi(t)|t|^{-1} \varphi(s)|s|^{-1}(\mu+1)\right. \\
\left.+\varphi(t) \varphi^{\prime}(s) t^{-1}+\varphi(s) \varphi^{\prime}(t) s^{-1}\right] d t d s .
\end{array}
$$


Интегрируя по частям, находим, что второе слагаемое равно нулю. Множество гладких функций с носителем в $\mathbf{R}^{1} \backslash\{0\}$ плотно в $H_{\mu}$, что доказывает унитарность $J_{\mu}^{*}$ в $H_{\mu}$. Утверждение 1 доказано.

Свойство (4) является ключевым для эффективного решения задач прогноза и канонического представления $\xi_{\mu}$ на отрезке $\Delta$. Оно позволяет путем дробно-линейной замены времени свести указанные задачи к полупрямой $(-\infty, 0)$. В силу стационарности $\xi_{\mu}$ эти задачи на полуоси элементарно решаются с помощью разложения Волда [20]. Чтобы вернуться к отрезку $\Delta$, надо использовать обратную замену времени. При этом отрезок $\Delta=\left[t_{0}, t_{1}\right]$ удобно рассматривать на расширенной ориентированной прямой, считая

$$
\Delta=\left[t_{0}, t_{1}\right]= \begin{cases}t_{0} \leqslant t \leqslant t_{1}, & t_{0}<t_{1} \\ {\left[t_{0}, \infty\right) \cup\left(-\infty, t_{1}\right),} & t_{0}>t_{1}\end{cases}
$$

т.е. $\left[t_{0}, t_{1}\right] \cup\left(t_{1}, t_{0}\right)=\mathbf{R}^{1} \cup\{\infty\}$. Тогда мы получаем единый подход к указанным задачам на любом связном множестве расширенной прямой. В частности, задача прогноза $\xi_{\mu}$ по данным на отрезке $\left[t_{0}, t_{1}\right]$ при $t_{0}>t_{1}$ превращается в задачу интерполяции $\xi_{\mu}(t)$ с $t \in\left(t_{1}, t_{0}\right)$. Последнее означает прогноз $\xi_{\mu}(\varphi)$, supp $\varphi \subset\left(t_{1}, t_{0}\right)$, по данным $\left\{\xi_{\mu}(\varphi)\right.$, supp $\left.\varphi \in\left(t_{1}, t_{0}\right)\right\}$. Сигма-алгебру событий, порожденных $\left\{\xi_{\mu}(\varphi)\right.$, supp $\left.\varphi \in(a, b)\right\}$, будем обозначать $\mathscr{F}_{a}^{b}\left(\xi_{\mu}\right)$.

2.2. Каноническое представление $\xi_{\mu}$. Следуя Крамеру, под каноническим представлением гауссовского процесса $\xi(t)$ через белый шум $b(t)$ будем понимать линейную связь $\xi=T b$, для которой фильтрации $\mathscr{F}_{t_{0}}^{t}(\xi)$ и $\mathscr{F}_{t_{0}}^{t}(b)$ совпадают для всех $t$. При рассмотрении $\xi_{\mu}$ на расширенной ориентированной прямой $\mathbf{R}^{1} \cup\{\infty\}$ параметр $t$ изменяется от $t_{0}$ до $\infty$ и далее от $-\infty$ до $t_{0}$. Рассмотрим операцию дробного интегрирования $I_{t_{0}}^{\alpha}$ порядка $\alpha>0$ с начальной точкой интегрирования $t_{0}$, т.е.

$$
I_{t_{0}}^{\alpha} f(t)=\int_{\left[t_{0}, t\right]} \frac{|t-s|^{\alpha-1}}{\Gamma(\alpha)} f(s) d s
$$

где область интегрирования понимается в смысле (7). Если $t_{0}=-\infty$, операция (8) известна как дробный интеграл Вейля, а при $-\infty<$ $t_{0}<t-$ как интеграл Римана-Лиувилля. В условиях $t_{0}<t$ дробный интеграл обладает групповым свойством в пространстве обобшенных функций с носителем в $\left[t_{0}, \infty\right): I^{\alpha} I^{\beta}=I^{\alpha+\beta}$, при этом $I^{-k+\beta}=D^{k} I^{\beta}$, $\beta \geqslant 0$, где $D=d / d t,[19]$.

Утверждение 2. Прочесс $\xi_{\mu},|\mu|<1$, допускает следуюшее каноническое представление через бельй шум:

$$
\xi_{\mu}(t)=(2 \pi)^{1 / 2}\left|t-t_{0}\right|^{\mu / 2} I_{t_{0}}^{\mu / 2}\left\{\left|s-t_{0}\right|^{-\mu / 2} b(s)\right\}(t) .
$$


При $\mu>0$ представление верно на расширенной прямой $\left[t_{0}, \infty\right) \cup$ $\left(-\infty, t_{0}\right)$, а при $\mu<0$ на полупрямой $\left[t_{0}, \infty\right)$. Обратная связь $\xi_{\mu}$ и аналогична:

$$
b(t)=(2 \pi)^{-1 / 2}\left|t-t_{0}\right|^{\mu / 2} I_{t_{0}}^{-\mu / 2}\left\{\left|s-t_{0}\right|^{-\mu / 2} \xi_{\mu}(s)\right\}(t) .
$$

$\Pi p u \mu>0 \quad t \in\left(t_{0}, \infty\right)$, a npu $\mu<0 \quad t \in\left[t_{0}, \infty\right) \cup\left(-\infty, t_{0}\right)$.

Д о к а з а т е л ь с т в о. Каноническое представление стационарного процесса $\xi$ на всей оси эквивалентно разложению Волда. Алгоритм получения этого разложения для обобщенных процессов со спектром степенного роста тот же, что и для обычных процессов, [20].

Процесс $\xi_{\mu}$ линейно регулярен, поскольку $|\ln f(x)|\left(1+\lambda^{2}\right)^{-1} \in L^{1}$, где $f(\lambda)=|\lambda|^{-\mu}$ - спектр $\xi_{\mu}$. Спектральная функция $|\lambda|^{-\mu}$ цопускает следуюцую факторизацию: $|\lambda|^{-\mu}=(\lambda+i 0)^{-\mu / 2}(\lambda-i 0)^{-\mu / 2}$, где $(\lambda-i 0)^{-\mu / 2}$ - след на вещественной оси функции $z^{-\mu / 2}$, аналитической в комплексной плоскости с разрезом $\operatorname{Im} z>0$ и вещественной при $z>0$. Комплексно-сопряженная функция к $z^{-\mu / 2}$ дает след на вещественной оси $(\lambda+i 0)^{-\mu / 2}$. Преобразование Фурье обобщенной функции $(\lambda-i 0)^{-\mu / 2}$ хорошо известно (см. [19]): $\left[(\lambda-i 0)^{-\mu / 2}\right]^{\wedge}(t)=2 \pi[\Gamma(\mu / 2)]^{-1} e^{-i \pi \mu / 4} t_{+}^{\mu / 2-1}$, где $t_{+}^{\alpha}=|t|^{\alpha} \mathbf{1}_{t>0}$. Отсюда $\xi_{\mu}(t)=(2 \pi)^{1 / 2} t_{+}^{\mu / 2-1} * b(t)[\Gamma(\mu / 2)]^{-1}$, где * означает свертку. Следовательно,

$$
\begin{aligned}
\xi_{\mu}(t) & =(2 \pi)^{1 / 2} I_{-\infty}^{\mu / 2} b(t), \\
b(t) & =(2 \pi)^{-1 / 2} I_{-\infty}^{-\mu / 2} \xi_{\mu}(t) .
\end{aligned}
$$

Последнее равенство следует из того, что операция дробного интегрирования Вейля образует однопараметрическую группу. Подставляя (5) в (11), имеем

$$
J_{\mu} \xi_{\mu}:=\xi_{\mu}\left(t^{-1}\right)|t|^{\mu-1}=(2 \pi)^{1 / 2} \int_{-\infty}^{t} \frac{(t-s)^{\mu / 2-1}}{\Gamma(\mu / 2)}\left(b\left(s^{-1}\right)|s|^{-1}\right) d s .
$$

Каноническое представление $\xi_{\mu}$ на прямой $(0, \infty) \cup(-\infty, 0)$ получается при $\mu>0$ отсюда формальной заменой переменных $t^{-1} \rightarrow t, s^{-1} \rightarrow s$. При этом надо различать два случая: $t>0$ и $t<0$. При строгом выводе последнее равенство надо интерпретировать в слабом смысле: $\xi\left(J_{\mu}^{*} \varphi\right)=(2 \pi)^{1 / 2} b\left(J_{0}^{*}\left(I_{-\infty}^{\mu / 2}\right)^{*} \varphi\right), \varphi \in K$, где * - символ сопряженной операции. В силу (5) $J_{\mu}^{*}=\left(J_{\mu}^{*}\right)^{-1}$. Поэтому $\xi(\varphi)=(2 \pi)^{1 / 2} b\left(J_{0}^{*}\left(I_{-\infty}^{\mu / 2}\right)^{*} J_{\mu}^{*} \varphi\right)$. Остается удостовериться, что $J_{0}^{*}\left(I_{-\infty}^{\mu / 2}\right)^{*} J_{\mu}^{*}=|t|^{-\mu / 2}\left(I_{0}^{\mu / 2}\right)^{*}|t|^{\mu / 2}$. Аналогично показывается, что из (12) при $\mu<0$ следует (10). В свою очередь (10) влечет (9) с $\mu<0$ и $t>t_{0}$, поскольку операция дробного интегрирования Лиувилля обладает групповым свойством на полуоси. Аналогично (9) влечет (10) для $\mu>0$ и $t>t_{0}$. 
2.3. Прогнозирование $\xi_{\mu}$. Обобщенный процесс $\left(\pi_{t_{0}}^{t_{1}} \xi_{\mu}\right)(\varphi)=$ $\mathbf{E}\left\{\xi_{\mu}(\varphi) \mid \mathscr{F}_{t_{0}}^{t_{1}}\left(\xi_{\mu}\right)\right\}, \varphi \in K$, будем называть прогнозом $\xi_{\mu}$ по данным в интервале $\left(t_{0}, t_{1}\right)$ расширенной прямой.

Утверждение 3. Прогноз $\xi_{\mu},|\mu|<1$, по данным $8\left(t_{0}, t_{1}\right)$ eсть обычный прочесс в дополнительном интервале, $\left(t_{1}, t_{0}\right)$, и имеет вид

$\pi_{t_{0}}^{t_{1}} \xi_{\mu}(t)=\frac{\sin (\pi \mu / 2)}{\pi} \int_{\left(t_{0}, t_{1}\right)}\left[\frac{\left(t-t_{1}\right)\left(t-t_{0}\right)}{\left(t_{1}-s\right)\left(s-t_{0}\right)}\right]^{\mu / 2} \frac{\bar{\xi}_{\mu}(d s)}{|t-s|}, \quad t \in\left(t_{1}, t_{0}\right)$

где $\bar{\xi}_{\mu}(d s)=d \xi_{\mu}\left(\chi_{[0, s]}\right)$ и интеграл понимается в среднем квадратичном.

Д о к а з а т е л ь с т в о. Найдем прогноз $\xi_{\mu}(t+\tau)$ в случае $\left(t_{0}, t_{1}\right)=$ $(-\infty, t)$. Из $(11),(12)$

$$
\begin{aligned}
& \Gamma\left(\frac{\mu}{2}\right) \pi_{-\infty}^{t} \xi_{\mu}(t+\tau)=\left[(2 \pi)^{1 / 2}(t+\tau)_{+}^{\mu / 2-1} \theta(t)\right] * b(t) \\
& \quad=\left[(t+\tau)^{\mu / 2-1} \theta(t)\right] *\left(I_{-\infty}^{-\mu / 2} \xi_{\mu}(t)\right)=I_{-\infty}^{-\mu / 2}\left\{(s+\tau)^{\mu / 2-1} \theta(s)\right\}(t) * \xi_{\mu}(t) .
\end{aligned}
$$

Здесь $\theta(t)=\mathbf{1}_{t>0} ;$ в последнем равенстве была использована коммутативность свертки. Первый элемент свертки в последнем равенстве легко вычисляется при $-1<\mu<0$ после замены переменных $(t-s)^{-1} \rightarrow s$; это дает

$$
\pi_{-\infty}^{t} \xi_{\mu}(t+\tau)=\frac{\sin (\pi \mu / 2)}{\pi}\left[\left(\frac{\tau}{t}\right)^{\mu / 2}(t+\tau)^{-1}\right] * \xi_{\mu}(t)
$$

В случае $\mu>0$ вычисления аналогичны; надо учесть только, что $I_{\infty}^{-\mu / 2}=$ $D I_{\infty}^{1-\mu / 2}$ и использовать разложение $(s+\tau)^{\mu / 2-1}=(s+\tau)^{\mu / 2-2}(t+\tau)-$ $(s+\tau)^{\mu / 2-2}(t-s)$.

В интеграле (14) расходимости могут возникать в окрестности точек $t$ и $-\infty$. Но в точке $t$ особенность имеет тип

$$
(t-s)^{-\mu / 2}\left(\bar{\xi}_{\mu}(s)-\bar{\xi}_{\mu}(t)\right)=|t-s|^{-H+1 / 2} O\left(|t-s|^{H-\varepsilon}\right)=O\left(|t-s|^{1 / 2-\varepsilon}\right),
$$

где $\varepsilon>0$ - лююбое малое число.

В окрестности $\infty$ интегральный хвост в свертке (14) имеет тип $I=\int_{-\infty}^{1} s^{-1-\mu / 2} \bar{\xi}_{\mu}(d s)$. В силу (5)

$$
I \stackrel{\mathrm{d}}{=} \int_{0}^{1} s^{1+\mu / 2} s^{-\mu-1} \bar{\xi}_{\mu}(d s)=\int_{0}^{1} s^{-\mu / 2} \bar{\xi}_{\mu}(d s) .
$$

Но, как мы уже показали, этот интеграл конечен.

Общая формула прогноза получается из (14) применением операций инверсии (5) и сдвига. Продемонстрируем это, используя формальную замену переменных. 
Если $J_{\mu}$ - операция инверсии (5), то из (14) для $t>t_{1}$ имеем

$$
J_{\mu} \pi_{-\infty}^{t_{1}} \xi_{\mu}(t)=\frac{\sin (\pi \mu / 2)}{\pi} \int_{(-\infty, t)}\left[\frac{t-t_{1}}{t_{1}-s}\right]^{\mu / 2} \frac{J_{\mu} \xi_{\mu}(s)}{|t-s|} d s
$$

или

$$
\pi_{t_{1}^{-1}}^{0} \xi_{\mu}\left(\frac{1}{t}\right)=\frac{\sin (\pi \mu / 2)}{\pi} \int_{(-\infty, t)}\left[\frac{t-t_{1}}{t_{1}-s}\right]^{\mu / 2}\left|\frac{s}{t}\right|^{\mu-1} \frac{\xi_{\mu}(1 / s)}{|t-s|} d s .
$$

Замена переменных $t_{1}^{-1}=t_{0}, t \rightarrow t^{-1}, s \rightarrow s^{-1}$ приводит нас $\mathrm{k}$ формуле (13) с $t_{1}=0$.

\section{3. Дробное броуновское движение}

Приведем следствия для $\mathrm{fBm}$, вытекающие из предыдущих утверждений. По определению

$$
w_{H}(t)=c_{H}^{-1 / 2} \xi_{\mu}\left(\chi_{(0, t)}\right), \quad \mu=2 H-1 .
$$

Отсюда следует равенство фильтраций $\mathscr{F}_{0}^{t}\left(w_{H}\right)=\mathscr{F}_{0}^{t}\left(\xi_{H}\right), t \in(0, \infty)$, и, в частности, тривиальность предельной $\sigma$-алгебры $\bigcap_{t \downarrow 0} \mathscr{F}_{0}^{t}\left(w_{H}\right)$. В противном случае будет нетривиальна аналогичная $\sigma$-алгебра для $\xi_{\mu}$, а в силу (5) и $\sigma$-алгебра $\bigcap_{t \downarrow-\infty} \mathscr{F}_{-\infty}^{t}\left(\xi_{\mu}\right)$. Последнее противоречит линейной регулярности $\xi_{\mu}$.

Утверждение 2 с учетом (15) дает связь $w_{H}$ с белым шумом $d w$.

Утверждение 4. а) Имеет место соотношение

$$
w_{H}(t)=\frac{\left(2 \pi / c_{H}\right)^{1 / 2}}{\Gamma(H-1 / 2)} \int_{0}^{t} K_{H}(t, s) d w(s), \quad 0<t<\infty
$$

əde

$$
\begin{aligned}
K_{H}(t, s) & =\int_{s}^{t}(\tau-s)^{H-3 / 2}\left[\left(\frac{\tau}{s}\right)^{H-1 / 2}-1\right] d \tau+\frac{(t-s)^{H-1 / 2}}{H-1 / 2} \\
& =O\left((t-s)^{H-1 / 2}\left(\frac{t}{s}\right)^{|H-1 / 2|}\right), \quad 0<s<t<t_{0}
\end{aligned}
$$

б) Гауссовский прочесс

$$
M_{H}(t)=\int_{0}^{t}[(t-s) s]^{1 / 2-H} d w_{H}(s), \quad t>0
$$

является мартингалом относительно естественной фильтраиии, порожденной прочессом $w_{H}, u$

$$
\mathbf{E}\left|d M_{H}(t)\right|^{2}=c|t|^{1-2 H} d t, \quad c=2 \pi \Gamma^{2}(3 / 2-H) c_{H}^{-1} .
$$


При $H<\frac{1}{2}$ независимье приращения $M_{H}$ можно доопределить при $t<0$ :

$$
\begin{aligned}
M_{H}(s)-M_{H}(t)=\int( & ((x-t) x]_{+}^{1 / 2-H} \\
& \left.\quad-[(x-s) x]_{+}^{1 / 2-H}\right) \operatorname{sign} x d w_{H}(x), \quad t, s<0 .
\end{aligned}
$$

При этом сохраняется свойство (19) и равенство фильтраиий $\mathscr{F}_{0}^{t}\left(d M_{H}\right)=\mathscr{F}_{0}^{t}\left(w_{H}\right)$ для всех $t$ расширенной прямой $[0, \infty) \cup(-\infty, 0)$.

Д ок а з а т е л ь с в о. а) Оценка (17) очевидна после замен переменных $\tau=s+x(t-s)$ при $s \rightarrow t$ и $\tau=x s$ при $s \rightarrow 0$; она доказывает существование стохастического интеграла в правой части (16) и равенство (16) при $t=0$. В силу (9) достаточно проверить теперь, что

$$
\left(H-\frac{1}{2}\right) \int_{0}^{\infty} \varphi^{\prime}(t) K_{H}(t, s) d s=\int_{0}^{\infty}(t-s)_{+}^{H-1 / 2}\left[\left(\frac{t}{s}\right)^{H-1 / 2} \varphi(t)\right]^{\prime} d t
$$

для любой гладкой финитной $\varphi$ на $(0, \infty)$. Если из обеих частей этого соотношения вычесть $\int_{0}^{\infty}(t-s)_{+}^{H-1 / 2}(t / s)^{H-1 / 2} \varphi^{\prime}(t) d t$, то оно проверяется интегрированием по частям левой части.

б) Перепишем (10) в форме

$$
\left(2 \pi c_{H}^{-1}\right)^{1 / 2}|t|^{1 / 2-H} w^{\prime}(t)=I_{0}^{(1 / 2-H)}\left(|s|^{1 / 2-H} w_{H}^{\prime}(s)\right)
$$

и найдем значения этих обобщенных процессов на характеристической функции $\chi_{(0, t)}, t>0$, при $0<H<1$ и на $\chi_{(t, s)}, t<s<0$, при $H<\frac{1}{2}$. В обоих случаях учтем, что $I^{\alpha}=D I^{(1+\alpha)}$ для интегралов Вейля и Лиувилля. Получим (18)-(20). Утверждение 4 доказано.

Взаимная плотность мер, отвечающих гауссовским процессам $x(t)+\varphi(t)$ и $x(t)$ на отрезке $\Delta$, где $\varphi$ - неслучайная функция, эффективно решается в терминах метрики пространства $H_{B}(\Delta)$ с воспроизводяцим ядром $B(t, s)=\mathbf{E} x(t) x(s),[21]$. Часто плотность называют формулой Гирсанова.

Пространство $H_{B}(\Delta)$ состоит из замыкания множества функций $\varphi_{\eta}(t)=\mathbf{E} x(t) \eta$, где $\eta$ принадлежит линейной оболочке $\{x(t), t \in \Delta\}$, по норме $\|\varphi\|_{B}=\mathbf{E}|\eta|^{2}$. Линейная связь $b=A w_{H}^{\prime}$ белого шума с $w_{H}$, см. (10), в которой $\mathscr{F}_{0}^{t}(b)=\mathscr{F}_{0}^{t}\left(w_{H}\right)$, немедленно решает вопрос о метрике для ядра $\mathbf{E} w_{H}(t) w_{H}(s)$ на отрезке $\Delta=(0, T)$, поскольку оператор

$$
A: H_{B}(\Delta) \ni \varphi \longrightarrow\left(\frac{c_{H}}{2 \pi}\right)^{1 / 2}|t|^{H-1 / 2} I_{0}^{1 / 2-H}\left(|s|^{1 / 2-H} \varphi^{\prime}\right) \in L^{2}(\Delta)
$$

устанавливает изометрию пространств $H_{B}(\Delta)$ и $L_{2}(\Delta)$. Отсюда имеем 
Утверждение 5. Пространство, воспроизводимое ядром

$$
B(t, s)=\frac{|t|^{2 H}+|s|^{2 H}-|t-s|^{2 H}}{2}
$$

на отрезке $(0, T)$, обладает метрикой

$$
\|m\|_{T}^{2}=\frac{c_{H}}{2 \pi} \int_{0}^{T}|\widetilde{m}(t)|^{2}|t|^{2 H-1} d t
$$

2de

$$
\widetilde{m}(t)=\frac{d}{d t} \int_{0}^{t} \frac{(t-s)^{1 / 2-H}}{\Gamma(3 / 2-H)} s^{1 / 2-H} m^{\prime}(s) d s .
$$

Пространство получается замыканием гладких функиий $m(t)$, $m(0)=0$, в указанной метрике.

Утверждение 6. Прогноз $w_{H}(t), t>t_{0}, 0<H<1$, по данным на отрезке $\left[0, t_{0}\right]$ есть

$$
\begin{aligned}
\pi_{0}^{t_{0}} w_{H}\left(k t_{0}\right)= & c \int_{0}^{1}\left[w_{H}\left(t_{0} s\right)-s^{2 H} w_{H}\left(t_{0}\right)\right]\left(\frac{(k-1) k}{(1-s) s}\right)^{H+1 / 2} \\
& \times\left((k-s)^{-1}-a(k)\right) d s \\
& +\left(k^{2 H}-(k(k-1))^{H+1 / 2} a(k)\right) w_{H}\left(t_{0}\right)
\end{aligned}
$$

где $k>1, c=\pi^{-1} \cos \pi H, a(k)=2 H\left(H+\frac{1}{2}\right)^{-1} k^{-1} F\left(1, \frac{1}{2}-H, H+\frac{3}{2} ; 1-k^{-1}\right)$ u $F$ - гипергеометрическая функиия. При $0<H<\frac{1}{2}$ формула (21) упрощается, поскольку можно формально положить $w_{H}\left(t_{0}\right)=0$.

3 а м е ч а н и е. Утверждение 6 является модификацией прогнозной формулы Григорьева [12], найденной для произвольного отрезка $\Delta$ и $0<H<\frac{1}{2}$. Заново предложенные решения задачи прогноза в [15] содержат $w_{H}$ в цифференциальной форме и сложное ядро линейного преобразования $d w_{H}$, см. (22) ниже. Регуляризация (21) в виде членов, включающих $w_{H}\left(t_{0}\right)$, распространяет формулу Григорьева на все $H$.

Доказательство. Из (15) имеем $w_{H}(t)=w_{H}\left(t_{0}\right)+$ $c_{H}^{-0.5} \xi_{\mu}\left(\chi_{\left(t_{0}, t\right)}\right), \mu=2 H-1$ и $\mathscr{F}_{0}^{t_{0}}\left(w_{H}\right)=\mathscr{F}_{0}^{t_{0}}\left(\xi_{\mu}\right)$. Поэтому

$$
\pi_{0}^{t_{0}} w_{H}(t)=w_{H}\left(t_{0}\right)+c_{H}^{-0.5} \pi_{0}^{t_{0}} \xi_{\mu}\left(\chi_{\left(t_{0}, t\right)}\right)
$$

В силу (13) имеем

$$
\pi_{0}^{t_{0}} w_{H}(t)=w_{H}\left(t_{0}\right)-\int_{0}^{1} K(k, s) d w_{H}\left(s t_{0}\right),
$$

где

$$
K(k, s)=\frac{\cos \pi H}{\pi} \int_{1}^{k}\left[\frac{(\tau-1) \tau}{(1-s) s}\right]^{H-1 / 2} \frac{d \tau}{\tau-s}
$$


Нетрудно видеть, что в области $T^{-1}<t_{0}<t<T, 0<s<t_{0}$ при $H \neq \frac{1}{2} K(t, s) s^{H-1 / 2}$ имеет порядок $o(1)$, если $t-t_{0}=o\left(t_{0}-s\right)$, и $\left[\left(t-t_{0}\right) /\left(t_{0}-s\right)\right]^{(H-1 / 2)+}$ в противном случае. Остается проинтегрировать полученное соотношение по частям. Все формальные действия будут законными при всех $H$, если использовать следующую регуляризацию: вместо $d w_{H}\left(s t_{0}\right)$ подставить $d\left[w_{H}\left(s t_{0}\right)-s^{2 H} w_{H}\left(t_{0}\right)\right]$. Но тогда надо изменить коэффициент при $w_{H}\left(t_{0}\right)$. Окончательный результат приведен в (21). Его легко проверить, сравнив производные по $k$ для выражения (21) и регуляризованного (22); при этом надо учесть, что $\left[(k(k-1))^{H+1 / 2} a(k)\right]^{\prime}=2 H(k(k-1))^{H-1 / 2}$. Утверждение 6 доказано.

Преобразование $x_{H}(t)=e^{-H t} w_{H}\left(e^{t}\right)$ переводит $w_{H}(t), t>0$, в стационарный процесс, который по аналогии со случаем $H=\frac{1}{2}$ можно назвать дробным процессом Орнштейна-Уленбека. Каноническое представление $(17)$ для $w_{H}(t), t>0$, легко трансформируется в разложение Волда для $x_{H}$. Отсюда с помощью преобразования Фурье получаем

Утверждение 7. Прочесс $x_{H}(t)$ имеет спектральную функиию

$$
f_{H}(\lambda)=\frac{2 \pi}{c_{H}}\left|\frac{\Gamma(-H-i \lambda)}{\Gamma\left(\frac{1}{2}-i \lambda\right)}\right|^{2}=O\left(|\lambda|^{-2 H-1}\right), \quad|\lambda| \rightarrow \infty .
$$

Линейная регулярность $x_{H}(t)$ является источником другого доказательства закона $0-1$ для событий $\bigcap_{t \downarrow 0^{\circ}} \mathscr{F}_{0}^{t}\left(w_{H}\right)$.

Линейное неупреждающее преобразование $w_{H}(t)$ со спектральной характеристикой $\varphi(\lambda)=1-2 H /(H-i \lambda),|\varphi(\lambda)|=1$, не меняет спектра гауссовского процесса $x_{H}(t)$ и, следовательно, не меняет его конечномерные распределения. Если перевести это замечание в термины $w_{H}$, то получим утверждение, впервые отмеченное в [22] для $H=\frac{1}{2}$.

Утверждение 8. На полупрямой $t>0$

$$
\widetilde{w}_{H}(t)=w_{H}(t)-2 H \int_{0}^{t} w_{H}(s) s^{-1} d s
$$

является FBM-прочессом и $\mathscr{F}_{0}^{T}\left(\widetilde{w}_{H}\right)=\mathscr{F}_{0}^{T}\left(M_{H}(t)-t / T \eta_{T}\right)$, аде $\eta_{T}=$ $2 H \int_{0}^{T}(s / T)^{2 H-1} d M_{H}(s)$ не зависит от $\mathscr{F}_{0}^{T}\left(\widetilde{w}_{H}\right)$. При $H=\frac{1}{2}$ имеем $\eta_{t}=M_{H}(t)=w_{H}(t)$.

И. Норрос и Е. Валкейла стимулировали написание данной заметки, за что автор им глубоко признателен.

\section{СПИСОК ЛИТЕРАТУРЫ}

1. Крейн М.Г. Об одном новом методе решения линейных интегральных уравнений первого и второго рода. - Докл. АН СССР, 1955, т. 100, № 3, с. 413-416.

2. Гохберг И. ЦІ., Крейн М.Г. Теория вольтерровых операторов в гильбертовом пространстве и ее приложения. М.: Наука, 1967, 508 с. 
3. Молчан Г. М. Гауссовские процессы с асимптотически степенным спектром. Теория вероятн. и ее примен., 1969 , т. 14 , в. 3 , с. $556-559$.

4. Колмогоров А. Н. Кривые в гильбертовом пространстве, инвариантные по отношению к однопараметрической группе движений. - Докл. АН СССР, 1940, т. 26, № 1, c. $6-9$.

5. Колмогоров А.Н. Спираль Винера и некоторые цругие интересные кривые в гильбертовом пространстве. - Докл. АН СССР, 1940, т. 26, № 2, с. 115-118.

6. Яглом А. М., Пинскер М. С. Случайные процессы со стационарными приращениями $n$-го порядка. - Докл. АН СССР, 1953, т. 90, №5, с. 731-734.

7. Пинскер M. С., Яглом A.M. О линейном экстраполировании случайных процессов со стационарными n-ми приращениями. - Докл. АН СССР, 1954, т. 94, № 3 , c. $385-388$.

8. Ito K. Stationary random distributions. - Mem. Coll. Sci. Univ. Kyoto, 1954, v. 28, p. 209-223.

9. Гельфанд И. М. Обобщенные случайные процессы. - Докл. АН СССР, 1955, T. 100 , № 5 , c. $853-856$.

10. Hunt G. A. Random Fourier transforms. - Trans. Amer. Math. Soc., 1951, v. 71, p. $38-69$.

11. Яглом A.M. Корреляционная теория процессов со случайными стационарными n-ми приращениями. - Матем. сб., 1955, т. 37, с. 141-196.

12. Григорьев C. В. Решение задач экстраполяции и интерполяции для одного нестационарного процесса. - Уч. записки Казанского гос. ун-та, 1967, т. 127, № 3 , c. $49-61$.

13. Mandelbrot B. B., Van Ness J. W. Fractional Brownian motions, fractional noises and applications. - SIAM Rev., 1968, v. 10, № 4, p. 422-437.

14. Молчан Г. М., Голосов Ю. И. Гауссовские стационарные процессы с асимптотически степенным спектром. - Докл. АН СССР, 1969, т. 184, № 3, с. 546-549.

15. Gripenberg G., Norros I. On the prediction of fractional Brownian motion. - J. Appl. Probab., 1996, v. 33, № 2, p. 400-410.

16. Norros I., Valkeila E., Virtamo J. An elementary approach to a Girsanov formula and other analytical results on fractional Brownian motions. - Bernoulli, 1999, v. 5, № 4, p. 571-587.

17. Decreusefond L., Üstünel A.S. Fractional Brownian Motion: Theory and Applications. - ESAIM: Proceedings. Fractional Differential Systems: Models, Methods and Applications, 1998 , v. 5, p. 75-86.

18. Decreusefond L., Üstünel A.S. Stochastic analysis of the fractional Brownian motion. - Potential Anal., 1999, v. 10, № 2, p. 177-214.

19. Гельфанд И. М., ШІилов Г. Е. Обобщенные функции и действия над ними. М.: Физматгиз, 1958, 470 с.

20. Дуб Дж. Л. Вероятностные процессы. М.: ИЛ, 1956, 605 с.

21. Ибрагимов И. А., Розанов Ю. А. Гауссовские случайные процессы. М.: Наука, $1970,384 \mathrm{c}$.

22. Jeulin T., Yor M. Filtration des ponts browniens et équations différentielles stochastiques linéaires. - Lecture Notes in Math., 1990, v. 1426, p. 227-265.

Поступила в редакцию 8.IX.2000 